\title{
Insular and anterior cingulate cortex deep stimulation for central neuropathic pain
}

\author{
Disassembling the percept of pain
}

\begin{abstract}
Ricardo Galhardoni, Geront, DDS, PhD, Valquíria Aparecida da Silva, RN, Luís García-Larrea, MD, PhD, Camila Dale, PhD, Abrahão F. Baptista, BPhysio, PhD, Luciana Mendonça Barbosa, MD, Luciana Mendes Bahia Menezes, MD, Silvia R.D.T. de Siqueira, DDS, PhD, Fernanda Valério, MD, Jefferson Rosi, Jr., MD, Antonia Lilian de Lima Rodrigues, RN, Diego Toledo Reis Mendes Fernandes, MD, Priscila Mara Lorencini Selingardi, MSc, Marco Antônio Marcolin, MD, PhD, Fábio Luís de Souza Duran, PhD, Carla Rachel Ono, MD, PhD, Leandro Tavares Lucato, MD, PhD, Ana Mércia B. L. Fernandes, BPhysio, Fábio E. F. da Silva, MD, Lin T. Yeng, MD, PhD, André R. Brunoni, MD, PhD, Carlos A. Buchpiguel, MD, PhD, Manoel J. Teixeira, MD, PhD, and Daniel Ciampi de Andrade, MD, PhD
\end{abstract}

Neurology ${ }^{\circledR}$ 2019;92:e2165-e2175. doi:10.1212/WNL.0000000000007396

\section{Abstract}

\section{Objective}

To compare the analgesic effects of stimulation of the anterior cingulate cortex (ACC) or the posterior superior insula (PSI) against sham deep (d) repetitive (r) transcranial magnetic stimulation (TMS) in patients with central neuropathic pain (CNP) after stroke or spinal cord injury in a randomized, double-blinded, sham-controlled, 3-arm parallel study.

\section{Methods}

Participants were randomly allocated into the active PSI-rTMS, ACC-rTMS, sham-PSI-rTMS, or sham-ACC-rTMS arms. Stimulations were performed for 12 weeks, and a comprehensive clinical and pain assessment, psychophysics, and cortical excitability measurements were performed at baseline and during treatment. The main outcome of the study was pain intensity (numeric rating scale $[\mathrm{NRS}]$ ) after the last stimulation session.

\section{Results}

Ninety-eight patients (age $55.02 \pm 12.13$ years) completed the study. NRS score was not significantly different between groups at the end of the study. Active rTMS treatments had no significant effects on pain interference with daily activities, pain dimensions, neuropathic pain symptoms, mood, medication use, cortical excitability measurements, or quality of life. Heat pain threshold was significantly increased after treatment in the PSI-dTMS group from baseline (1.58, 95\% confidence interval $[\mathrm{CI}] 0.09-3.06])$ compared to sham-dTMS $(-1.02,95 \% \mathrm{CI}-2.10$ to $0.04, p=0.014)$, and ACC-dTMS caused a significant decrease in anxiety scores $(-2.96,95 \% \mathrm{CI}$ -4.1 to -1.7$])$ compared to sham-dTMS $(-0.78,95 \%$ CI -1.9 to $0.3 ; p=0.018)$.

\section{Conclusions}

ACC- and PSI-dTMS were not different from sham-dTMS for pain relief in CNP despite a significant antinociceptive effect after insular stimulation and anxiolytic effects of ACC-dTMS. These results showed that the different dimensions of pain can be modulated in humans noninvasively by directly stimulating deeper SNC cortical structures without necessarily affecting clinical pain per se.

\section{Correspondence}

Dr. Ciampi de Andrade

ciampi@usp.br

\section{ClinicalTrials.gov identifier: \\ NCT01932905.}




\section{Glossary}

ACC $=$ anterior cingulate cortex $\mathbf{C E}=$ cortical excitability; $\mathrm{CI}=$ confidence interval; $\mathrm{CNP}=$ central neuropathic pain; $\mathrm{CPSP}=$ central poststroke pain; dTMS = deep transcranial magnetic stimulation; NPSI = Neuropathic Pain Symptoms Inventory; NRS = numeric rating scale; PSI = posterior superior insula; $\mathbf{Q S T}=$ quantitative sensory testing; $R M T$ = rest motor threshold; rTMS = repetitive transcranial magnetic stimulation; $\mathbf{S C I}=$ spinal cord injury; TA $=$ tibialis anterior.

Central neuropathic pain (CNP) is caused by a lesion or disease of the somatosensory pathways in the CNS. CNP is a secondary complication of common diseases such as stroke (i.e., central poststroke pain $[\mathrm{CPSP}]$ ) and spinal cord injury (SCI) secondary to traumatic, inflammatory, or demyelinating diseases. CPSP occurs in $2 \%$ to $8 \%$ of stroke survivors and is present in up to $18 \%$ of those presenting with somatosensory deficits and up to $50 \%$ of those with lesions affecting solely the spinothalamic pathways. ${ }^{1}$ Pain also ranks among the most debilitating complications of traumatic $\mathrm{SCI}^{2}$ affecting $>80 \%$ of patients in the 5 years after trauma and leading to CNP in up to $59 \%$ of individuals. SCI can also be caused by inflammatory insults occurring in demyelinating disorders such as multiple sclerosis (MS) or neuromyelitis optica spectrum disorders. These conditions affect $>2$ million individuals globally, leading to a lifetime prevalence of CNP of at least $28 \%$.

Unfortunately, attempts to control CNP have been marked by refractoriness and unsuccessfulness. For instance, CPSP failed to respond satisfactorily to levetiraceta, ${ }^{3}$ pregabalin, ${ }^{4}$ duloxetine, ${ }^{5}$ morphine, ${ }^{6}$ and carbamazepine, ${ }^{7}$ while SCI-CNP has not responded to venlafaxine, ${ }^{8}$ levetiracetam, ${ }^{9}$ or dronabinol. ${ }^{10}$ MS-related CNP failed to respond to cannabinoids ${ }^{11}$ and duloxetine. ${ }^{5}$ In the rare existing positive trials, the magnitude of the analgesic effect was frequently small such as the response of MS-related CNP and CPSP/SCI to duloxetine or pregabalin ${ }^{12}$ or the response to opioids ${ }^{13}$ in SCI-CNP. In other instances, positive results were derived from very small studies. Thus, treatment of CNP remains a major unmet need and has been the focus of several new treatment options such as noninvasive neuromodulation.

Superficial repetitive ( $\mathrm{r}$ ) transcranial magnetic stimulation (TMS) has been shown to be potentially effective in the treatment of major depression and in stroke rehabilitation. Similarly, rTMS targeted to the primary motor cortex (M1) has been successfully used to relieve several chronic pain syndromes such as fibromyalgia, complex regional pain syndrome, and peripheral neuropathic pain. A large body of evidence indicates that M1 rTMS influences blood flow, neuronal hyperactivity, and neurotransmitter release in brain, brainstem, and spinal cord areas distant from M1 in both human patients and rodents. Several brain structures known to be engaged during the occurrence of chronic pain are also modulated by M1 stimulation. Nevertheless, a significant proportion of patients fail to respond to M1 stimulation, and the analgesic effects of superficial rTMS, regardless of the target chosen, have not been clearly demonstrated for patients with CNP. Studies including exclusively patients with CNP using superficial cortical targets such as the primary motor and prefrontal cortices were underpowered or negative or had short-lasting effects. ${ }^{14}$

The hallmark of CNP is the presence of pain with neuropathic descriptors in an area of impaired somatosensory function, frequently affecting thermal sensations. ${ }^{15}$ It has been proposed that a lesion to the spinothalamic projections would lead to plastic changes in brain areas implicated in the processing of pain; deafferentation of the insular recipients of these inputs, leading to insular functional disinhibition; and increased activity, causing increased processing of ascending inputs by mesial pain pathways, including those targeting the parabrachial nucleus and the anterior cingulate cortex $(\mathrm{ACC})^{16}$ and amygdala. While some aspects of this model have been challenged, the idea of an overactivation of these deep structures has been supported by functional brain imaging studies in normal humans in acute pain, as well as in patients with neuropathic pain. ${ }^{17}$ Similarly, functional connectivity studies ${ }^{18}$ have also reported a central role of these structures in instances of neuropathic pain. The possibility of targeting these deep structures to relieve pain has been tested recently. Insular stimulation in rats under a peripheral neuropathy model caused significant analgesic effects that depended on the availability of opioid and cannabinoid receptors, ${ }^{19}$ and high-frequency stimulation of the ACC by optogenetics influenced mood-related responses in rats. ${ }^{20}$ In recent years, technologic improvements have allowed the noninvasive modulation of deep cortical structures such as the dentate nucleus, the ACC, ${ }^{21}$ and the insula by deep rTMS in awake humans. Some preliminary results have proposed that these new approaches may have significant effects in mood disorders and experimental pain settings ${ }^{20}$ and could provide a short-cut approach to target deep cortical areas directly implicated in the processing of physiologic pain and known to have an abnormal functioning in CNP settings.

We hypothesized that noninvasive modulation of deep cortical structures known to be dysfunctional in CNP would provide analgesic effects in CNP. We compared the analgesic effects of deep rTMS to the ACC and posterior superior insula (PSI) with sham stimulation in patients with CNP who were refractory to first-, second-, and third-line drugs ${ }^{22}$ in a 12 week randomized, double-blind, controlled study.

\section{Methods}

The study was carried out at the Hospital das Clínicas of the University of São Paulo. Our Ethics Review Board approved 
the protocol (No. 690.455), and all patients provided written informed consent before inclusion in the study. This study was registered as NCT01932905 at clinicaltrials.org.

\section{Patients}

Patients were prospectively recruited from different outpatient clinics from our hospital complex by their respective physician, who had no other role in the study. Sociodemographic and clinical evaluations were performed in all participants. Patients with chronic ( $>3$ months) defined CNP due to stroke or spinal cord lesions were included. Stroke patients with lesions located on either the ACC or the right PSI were not included. Major psychiatric disorder, language and cognitive disturbances preventing pain assessment by questionnaires, and presence of seizures within the previous 6 months were also exclusion criteria.

\section{Study design}

This was a head-to-head double-blind, randomized, parallelgroup controlled trial to assess the efficacy of deep rTMS (dTMS) to the PSI (PSI-dTMS) against dTMS to the ACC (ACC-dTMS) compared to sham stimulation (sham-dTMS) in CNP due to stroke or SCI. The sham group received sham stimulation to either the ACC or PSI in a 1:1 proportion. Thus, participants were randomly allocated into the active PSI-dTMS, ACC-dTMS, sham-PSI-dTMS, or sham-ACCdTMS in a 2:2:1:1 ratio in blocks of 12 and were stimulated daily for 5 consecutive days and then weekly during maintenance sessions until the 12 th week (i.e., total of 16 stimulation sessions, figure e-1 available from Dryad, doi.org/10.5061/ dryad.630mg39). Randomization was performed with the use of electronic software (randomizer.com). Allocation concealment was granted by the use of password-protected files in a computer assessed by a research coordinator with no routine contact with patients or with other researchers during the data collection period. Pain assessments were performed at baseline; after the first week of treatment (induction period); and after the end of the first, second, and third months of treatment according to figure e-1 available from Dryad (doi.org/ 10.5061/dryad.630mg39) (study design). Psychophysics, cortical excitability (CE) measurements, mood, and qualityof-life assessments were performed at baseline and at the end of the study (i.e., right after the last dTMS stimulation). All clinical evaluations were performed by researchers with no other role in the study who were not implicated in patient stimulation, psychophysics, or neurophysiologic assessments and who worked outside the neuromodulation laboratory.

\section{Treatment session}

\section{Target localization and dTMS setup}

A 3-dimensional image of the head was obtained with volumetric T1-weighted MRI for frameless stereotaxic neuronavigation (Brainsight, Rogue Research, Montreal, Quebec, Canada) for all patients. The tibialis anterior (TA) muscle rest motor threshold was measured as a percentage of maximum stimulator output and was obtained for all participants. All stimulations were performed at $90 \%$ of TA rest motor threshold (RMT). For PSI determination and stimulation, the neuronavigation procedure was performed to ensure accurate positioning of the coil and, most important, the correct identification of the posterior and superior aspect of the PSI. ${ }^{23}$ PSI-dTMS and sham-PSI-dTMS treatment sessions were performed with a cooled DB-80 double-cone coil (Magventure Tonika-Elektronik, Farum, Denmark), which creates an induced electric current 4 to $5 \mathrm{~cm}$ underneath the center of the coil and has one of the best depth/focality relationship among several coils. ${ }^{24}$ During sessions, the coil was oriented at a tangent to the scalp, with the main phase of the induced current in the posterior-to-anterior direction. The stimulation session consisted of 15 series of 10 -second trains at $10 \mathrm{~Hz}$ and an intertrain interval of 50 seconds (total 1,500 pulses per session) ${ }^{23}$ For ACC determination and stimulation, dTMS to the ACC was performed with a Magstim Rapid ${ }^{2}$ (Magstim Co, Ltd, Whitland Carmarthenshire, UK) equipped with an $\mathrm{H}-6$ coil specifically designed to stimulate the ACC. The ACC was determined according to a technique described previously. Briefly, TA RMT was obtained as described above, and the $\mathrm{H}$ coil was moved forward $4 \mathrm{~cm}$ anterior to the motor hotspot. ${ }^{21}$ Sham stimulations were applied with an active figure-of- 8 coil placed above the unplugged double-cone coil as previously described ${ }^{25}$ for the insular stimulation. For the ACC, ${ }^{26-28}$ the built-in sham system was used. Participants were briefed that physical scalp and noise sensations would be emitted by all types of coils (sham and real).

Researchers who delivered dTMS were blinded to all other assessments except the type of TMS being applied and had no other role in the study. Care was taken not to set patients' appointments at the same time period so that waiting-room conversations could be avoided and to mitigate possible blinding integrity jeopardizing. Blinding assessment was performed at the end of the study by asking all participants direct questions about blinding.

\section{Pain and related factors assessment}

The study main outcome was pain intensity, as measured by an 11-point numeric rating scale (NRS) anchored at 0 (no pain) and 10 (most intense pain imaginable) from the "average pain intensity within the last 24 hours" item from the brief pain inventory. Secondary outcomes were the global impression of change and the outcome measurements below:

1. Short-form McGill Pain Questionnaire;

2. Brief Pain Inventory short form, which includes pain severity index (mean of questions 3-6) and pain interference in daily activities (mean of questions 9A-9G, ranging from 0 to 70 , where 70 indicates the maximal pain interference possible);

3. Douleur Neuropathique- 4 to screen for neuropathic pain, being positive for scores $\geq 4$;

4. Neuropathic Pain Symptoms Inventory (NPSI), which provides characterization of neuropathic pain symptoms in 5 domains (spontaneous superficial and deep pain, paroxysmal pain, evoked pain, and paresthesia/ 
dysesthesia) and its temporal pattern. ${ }^{29}$ It is rated on a range from 0 to 100 , and higher scores denote more intense neuropathic pain-related symptoms;

5. Hospital Anxiety and Depression Scale, which assesses mood symptoms (self-administered 21 items). Scores range from 0 to 21 for anxiety and depression subscores, and higher scores denote more intense mood symptoms; and

6. 12-Item Short-Form Survey, which scores the main domains of health-related quality of life and provides a final total score.

We also performed exploratory assessments to assess whether medication use and etiology of CNP (SCI or CPSP) played a role in response to active treatments.

\section{Psychophysics testing}

Patients underwent a quantitative sensory testing (QST) battery intended to assess small fiber-mediated (A- $\partial, \mathrm{C})$ sensory inputs at site of more severe neuropathic pain. Thermal thresholds were measured with a TSA-2001 device (Medoc, Ramat Yshai, Israel), as described elsewhere. Thermal detection thresholds (warm detection, cold detection, cold pain, and heat pain thresholds) were determined as described elsewhere. ${ }^{30}$ Baseline pain body side, pain site, and their respective thermal values were compared between groups at baseline. Then, QST measurements changes obtained after the last dTMS session were compared between groups.

\section{Neurophysiologic assessment (CE measurements)}

Participants underwent a 30-minute experimental session for CE measurements with a MagPROX100 machine (Magventure Tonika Elektronic, Farum, Denmark) using a circular coil (C-100 Magventure Tonika Elektronic). RMT, motor evoked potentials, short-interval intracortical inhibition at interstimulus intervals of 2 and 4 milliseconds, and intracortical facilitation at interstimulus intervals of 10 and 15 milliseconds were done in both hemispheres as previously reported. ${ }^{31} \mathrm{We}$ looked for side differences within each study arm (i.e., PSIdTMS, ACC-dTMS, and respective shams) to assess right-left differences in $\mathrm{CE}$ values. Data were also compared according to changes after dTMS treatment (CE results from last session subtracted from baseline values) across groups. In addition, each single $\mathrm{CE}$ parameter was classified for each individual according to published normative data as normal, high (above the $95 \%$ confidence interval [CI]), or low (below $95 \% \mathrm{CI}$ ), and the corresponding percentages of these 3 possible outcomes were compared between groups. ${ }^{31}$

\section{Statistical methods}

The exploratory analysis initially evaluated distributions, frequencies, and percentages for each of the numeric and categorical variables. Categorical variables were evaluated for near-zero variation. Extensive graphical displays were used for both univariate analysis and bivariate associations. Missing data were explored with a combination of graphical displays involving univariate, bivariate, and multivariate methods. Missing data imputation was performed using a k-nearest neighbor algorithm $(\mathrm{n}=5)$. We assessed randomization effectiveness by evaluating balance regarding baseline variables, comparing the 2 interventions and the control arms. We also assessed whether the 2 sham groups were balanced at baseline and at the end of the study (results provided in tables e- 6 and e-7 available from Dryad, doi.org/10.5061/dryad.630mg39). Results from balancing were evaluated through 1-way analysis of variance and $\chi^{2}$ tests. According to these results, data from both sham arms were pooled together into a single sham arm for comparisons with PSI and ACC active dTMS arms as previously reported. ${ }^{32}$ To evaluate intervention efficacy (NRS), we performed a series of generalized linear models and generalized estimating equation models to evaluate the association between all previously reported outcomes in the 2 intervention arms and the sham. Generalized linear models take into account only the final measured outcome, while generalized estimating equations account for all intermediate follow-up measurements. Results were adjusted for age and sex and reported as predicted means for numeric outcomes and odds ratios for Boolean (yes/no) outcomes, along with $95 \%$ CIs. Given that the randomization did not present any methodologic flaws, we did not adjust outcome results for unbalanced variables. We followed an intention-totreat principle in our analysis, including participants regardless of their compliance to the trial protocol. All analyses were performed with the $\mathrm{R}$ statistical language and the ggplot $2^{33}$ and rmarkdown ${ }^{34}$ packages. Results were also expressed as average \pm SD. Generalized linear models were used for variable data collected only at baseline and at study termination. The primary outcome (pain intensity at the end of the study) was also compared between patients experiencing pain on the left and those with pain on the right side of the body, and the effects of the different treatment groups were compared between patients presenting left- or right-side-predominant pain. Because the Kolmogorov-Smirnov test revealed that catastrophism, mood, quality of life, QST, and CE values did not have a normal distribution, differences between groups were compared with nonparametric tests (Kruskal-Wallis test, followed by pairwise comparisons of change between independent groups (Mann-Whitney $U$ test). CE results of each individual were also further classified according normative data into normal, ${ }^{31}$ high, or low values based on $95 \%$ CIs and compared by the $\chi^{2}$ test. Sample size was calculated with G3 power software. Because this is the first dTMS trial aiming at deep cortical structures in CNP, we calculated an estimated sample size on the basis of the largest sham-controlled longterm study available to date using TMS for pain relief with induction and maintenance sessions. ${ }^{35}$ We used the effect size obtained at the end of the weekly maintenance sessions (0.66), and on the basis of a power of 0.90 and $\alpha$ error probability of 0.01 , the estimated sample size was 33 individuals per group. The effect size of the study was calculated from the effects of the $3 \mathrm{arms}$ on pain intensity.

\section{Data availability}

Anonymized data can be made available to qualified investigators on request to the corresponding author. 


\section{Results}

\section{Patients}

One hundred twenty-three patients were assessed for eligibility. One hundred patients (age 55.02 \pm 12.13 years, 45 female) were included and randomized. All included patients but 2 finished the study protocol, and all were included in the final analyses (figure e-2 available from Dryad, doi.org/10. 5061/dryad.630mg39). Baseline demographic characteristics (table e-1 available from Dryad), etiology of CNP, pain location, pain characteristics, mood, and quality-of-life results were similar between groups at baseline (table e-2 available from Dryad).

\section{Pain and related factors assessments}

The main outcome of the study (NRS score at week 12) was not significantly different between groups (figure, A) during the study. Given that the trial did not demonstrate a significant difference among intervention arms, we estimated the effect size and corresponding sample size that would be required to demonstrate a significant difference in case there was one. The effect size was 0.12 with a corresponding sample size of 272 participants per arm ( $n=816$ for the full trial) to demonstrate a conjectural difference while accounting for 3 hypotheses.

Similarly, active dTMS treatments had no significant effects on pain interference with daily activities (Brief Pain Inventory), pain dimensions (McGill Pain Questionnaire), neuropathic pain symptoms (NPSI), and number of medications used (table 1). A preplanned exploratory analysis of the effects of treatments on clusters of symptoms of neuropathic pain assessed by the NPSI showed that insular stimulation had a statistically significant increase in paroxysmal pain symptoms $(3.23,95 \%$ CI $2.27-4.18)$, compared to the sham group (1.4, 95\% CI 0.74-2.05). The percentage of responders who considered themselves as having improved or much improved on the Global Impression of Change was similar between groups and was not influenced by the etiology of CNP (CPSP vs SCI-related CNP) (table e-3 available from Dryad, doi.org/10.5061/dryad.630mg39). Catastrophizing (PCS) and quality of life (12-Item Short-Form Survey) were not significantly affected by either real dTMS or sham (table 2). Patients' and clinicians' Global Impression of Change

Figure Main study results
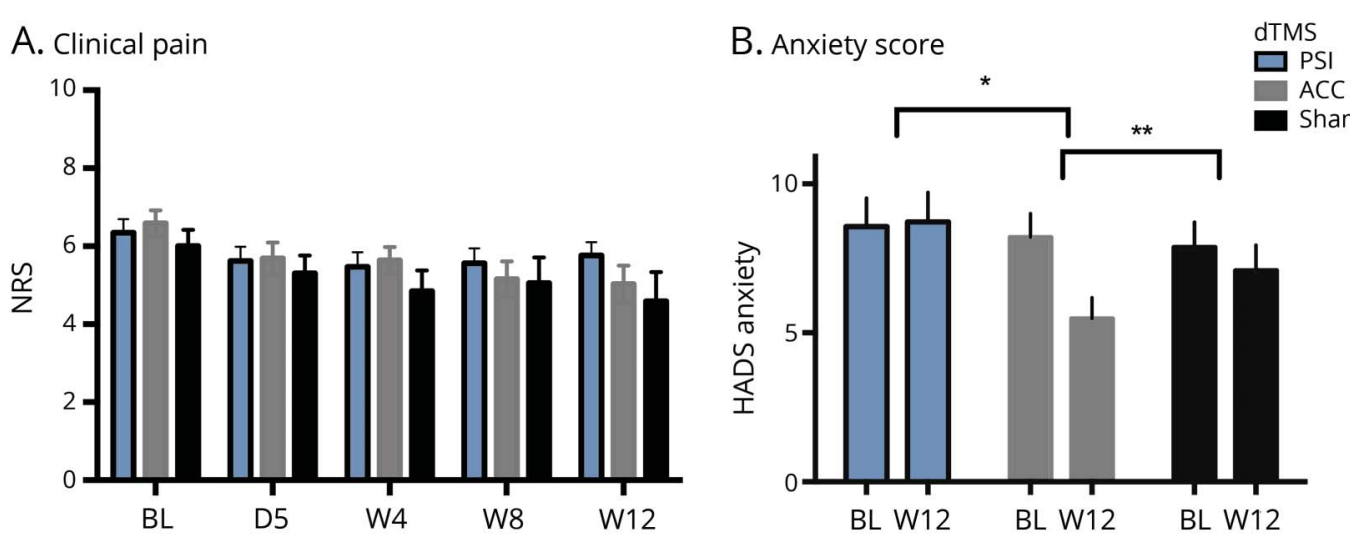

C.a. Heat pain threshold

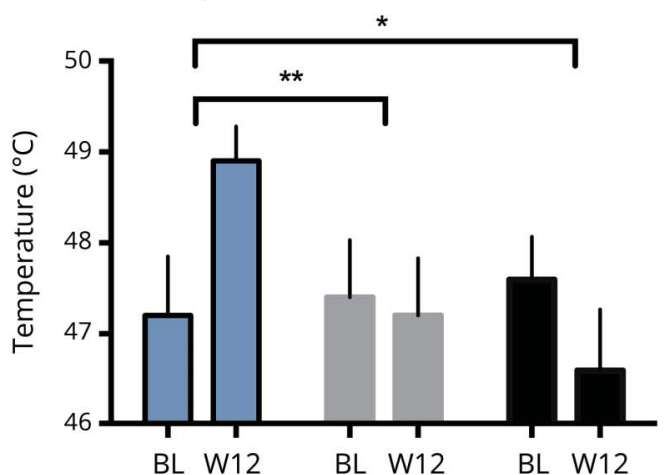

C.b. Warm detection threshold

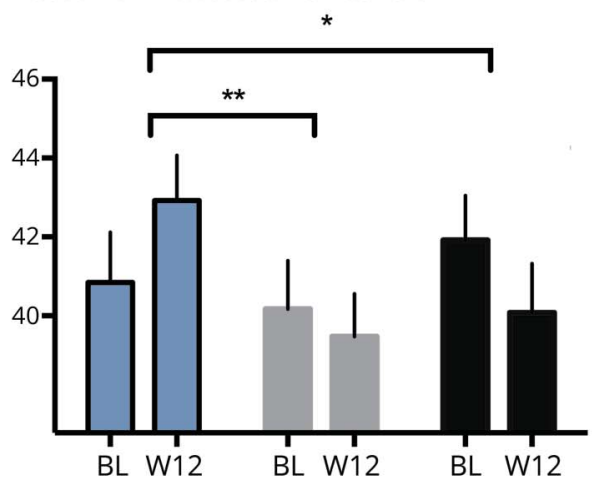

Study outline. (A) Pain intensity scores during the induction (day [D] 1-5) and maintenance (week [W] 2-8) periods. For the Numerical Rating Score (NRS), $0=$ no pain and $10=$ maximal pain imaginable. (B) Hospital Anxiety and Depression Scale (HADS) at baseline (BL) and at the end of the study (W12) in the 3 different groups. Comparisons between changes (W12 to baseline). Data are expressed as mean (SD) * $p=0.0001,{ }^{*} p=0.018$. (C.a) Heat pain threshold and (C.b) warm detection threshold at baseline and end of the study (W12) in the 3 different groups. Comparisons between changes (W12 to baseline). Heat pain threshold: * $p$ $=0.014,{ }^{\star \star} p=0.029$. Warm detection threshold: ${ }^{\star} p \leq 0.001,{ }^{\star}{ }^{*} p=0.006$. ACC $=$ anterior cingulate cortex; $\mathrm{dTMS}=\mathrm{deep}$ transcranial magnetic stimulation; PSI $=$ posterior superior insula. 
Table 1 Predicted means comparison between groups during the study

\begin{tabular}{llll}
\hline & dTMS-sham $(\mathbf{n}=\mathbf{3 2})$ & dTMS-ACC $(\mathbf{n}=\mathbf{3 3})$ & $\mathbf{d T M S}-\mathrm{PSI}(\mathbf{n}=\mathbf{3 3})$ \\
\hline BPI pain intensity score & $5.08(4.31-5.86)$ & $5.59(4.91-6.27)$ & $5.77(5.2-6.35)$ \\
\hline BPI pain interference daily activity score & $26.09(20.14-32.04)$ & $27.74(21.38 .34 .09)$ & $34.22(27.7-40.74)$ \\
\hline NPSI total score & $28.16(22.37-33.95)$ & $32.3(26.08-38.52)$ & $40.34(32.91-47.77)$ \\
\hline NPSI score: continuous ongoing pain cluster & $5.34(4.42-6.26)$ & $5.1(4.04-6.16)$ & $5.96(5.09-6.84)$ \\
\hline NPSI score: evoked pain cluster & $2.4(1.68-3.12)$ & $2.77(1.96-3.57)$ & $3.73(2.85-4.6)$ \\
\hline NPSI score: paroxysmal pain cluster & $1.4(0.74-2.05)^{a}$ & $2.34(1.72-2.96)$ & $3.23(2.27-4.18)^{a}$ \\
\hline NPSI score: burning pain cluster & $3.06(2.19-3.92)$ & $3.4(2.49-4.31)$ & $3.17(3.22-5.13)$ \\
\hline NPSI score: electric shocks cluster & $3.03(2.28-3.77)$ & $3.58(8.56-10.6)$ & $3.97(3.1-4.85)$ \\
\hline MPQ score & $8.93(7.96-9.9)$ & $0.9(0.74-1.07)$ & $10.61(9.38-11.84)$ \\
\hline Pain medications in use, $n$ & $0.92(0.68-1.16)$ & $0.89(0.69-1.08)$ \\
\hline
\end{tabular}

Abbreviations: ACC = anterior cingulate cortex; BPI = Brief Pain Inventory; dTMS = deep transcranial magnetic stimulation; MPQ = McGill Pain Questionnaire; $\mathrm{NPSI}=$ Neuropathic Pain Symptoms Inventory; PSI = posterior superior insula.

Values are presented as means from generalized estimating equations (95\% confidence interval $[\mathrm{Cl}]$ ).

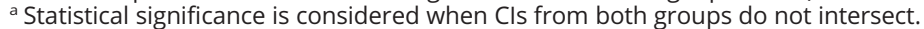

after treatment did not differ between groups (table e-3 available from Dryad). While depression did not significantly change after real dTMS treatment, the anxiety symptoms showed significant changes between groups $(p<0.001)$. ACC-dTMS caused a significant decrease in Hospital Anxiety and Depression Scale anxiety scores from baseline $(-2.96$, $95 \% \mathrm{CI}-4.1$ to -1.7$)$ compared to sham-dTMS $(-0.78,95 \%$ CI -1.9 to $0.3, p=0.018)$ and PSI-dTMS $(0.15,95 \% \mathrm{CI}-1.0$ to $1.3, p<0.001$ ) arms (figure, $\mathrm{B}$ and table 2). Concerning the main outcome measure, the 3 experimental treatments had similar effects on patients with right- or left-side-predominant CNP ( $p=0.276$ for right-sided pain, $p=0.350$ for left-sided pain). In a comparison of the treatment response between patients with right- and left-sided pain within each group, patients with left-sided pain presented results after insular $(p=$ $0.716)$ and ACC $(p=0.518)$ stimulation similar to those presenting with right-side-predominant pain. In addition, the different etiologies of CNP (i.e., CPSP, SCI) had similar response to deep rTMS in all 3 arms (table e-3 available from Dryad).

\section{Psychophysics}

All baseline QST measurements were similar between groups. Compared to baseline values, heat pain and warm detection thresholds were significantly higher on the painful side after PSI compared to sham-dTMS at the 12th week of treatment (figure, C.a and C.b). Differences in heat pain threshold were significantly different across groups $(p=0.026)$. Changes in heat pain threshold after treatment was significantly larger (i.e., increase in threshold) in the PSI-dTMS group (1.58, $95 \%$ CI $0.09-3.06)$ than in the sham $(-1.02,95 \%$ CI -2.10 to $0.04, p=0.014)$ and ACC-dTMS $(-0.16,95 \%$ CI -1.27 to $0.94, p=0.014)$ groups. No significant changes were seen after ACC simulation. Similarly, warm detection thresholds were significantly affected by insular stimulation $(p=0.001)$.
PSI-dTMS provided significantly larger increase in warm detection threshold $(2.07,95 \%$ CI $0.48-3.66, p=0.006)$ compared to ACC-dTMS $(-0.70,95 \%$ CI 0.44 to $-1.84, p<$ $0.001)$ and sham-dTMS $(-1.83,95 \%$ CI -0.57 to $-3.10, p=$ $0.006)$. Changes in cold pain and cool detection thresholds were not different between groups (table 2).

\section{Cortical excitability}

$\mathrm{CE}$ was measured bilaterally over the primary motor cortex. Normative data from healthy controls allowed us to classify each CE parameter as normal, high, or low on the basis of the 95\% CI (table e-4 available from Dryad, doi.org/10.5061/ dryad.630mg39). This revealed that patients with CNP from the 3 treatment arms had similar CE values, which were abnormal in $>80 \%$ of the individuals for nearly all measured parameters. In general, most motor evoked potential amplitudes tended to be low in patients with CNP. Paired-pulsebased responses such as intracortical inhibition were high (defective intracortical inhibition), while intracortical facilitation had a tendency to be show low results compared to reference data from healthy individuals. Despite this altered baseline profile, which was similar between groups, active dTMS of either the PSI or ACC caused no significant changes in $\mathrm{CE}$ measurements on the last day of stimulation, and no group differences were detected (table e-5 available from Dryad).

\section{Safety}

Adverse events were systematically assessed by a printed form at the end of each stimulation session. Pain (mostly headaches) after each dTMS was the most prevalent adverse event. The average pain score (NRS score 0-10) during the stimulation sessions was $2.0 \pm 3.4$ for the PSI group, $2.6 \pm 3.3$ for the ACC group, and $2.0 \pm 3.3$ for the sham-dTMS group. Other 
Table 2 Results from mood, catastrophizing, quality-of-life, and QST parameters for painful side at baseline and end of the study

\begin{tabular}{|c|c|c|c|c|c|c|c|c|c|c|}
\hline & \multicolumn{3}{|l|}{ Baseline } & \multicolumn{4}{|l|}{ W12 } & \multicolumn{3}{|l|}{$p$ Value } \\
\hline & PSI-dTMS & ACC-dTMS & Sham-dTMS & PSI-dTMS & ACC-dTMS & Sham-dTMS & $\begin{array}{l}\text { Change in score } \\
\text { between groups }\end{array}$ & $\begin{array}{l}\text { PSI-dTMS vs } \\
\text { sham-dTMS }^{b}\end{array}$ & $\begin{array}{l}\text { ACC-dTMS vs } \\
\text { sham-dTMS }^{\text {b }}\end{array}$ & $\begin{array}{l}\text { PSI-dTMS vs } \\
\text { ACC-dTMS }^{b}\end{array}$ \\
\hline \multicolumn{11}{|l|}{ HADS score } \\
\hline Anxiety & $8.5 \pm 5.4$ & $8.2 \pm 4.0$ & $7.8 \pm 4.7$ & $8.6 \pm 5.6$ & $5.5 \pm 4.2$ & $7.0 \pm 4.8$ & $0.001^{c}$ & 0.134 & $0.018^{b}$ & $0.0001^{\mathrm{b}}$ \\
\hline Depression & $8.4 \pm 5.1$ & $8.0 \pm 4.7$ & $7.3 \pm 4.1$ & $8.2 \pm 5.0$ & $6.4 \pm 5.5$ & $7.1 \pm 4.0$ & 0.913 & 0.684 & 0.793 & 0.782 \\
\hline Total & $16.9 \pm 10.3$ & $16,2 \pm 8.1$ & $15.2 \pm 8.1$ & $16.9 \pm 10.0$ & $12.0 \pm 9.1$ & $14.2 \pm 7.6$ & 0.939 & 0.916 & 0.814 & 0.916 \\
\hline PCS score & $28.1 \pm 15.5$ & $24.0 \pm 12.9$ & $25.4 \pm 13.0$ & $25.7 \pm 16.1$ & $17.5 \pm 14.6$ & $19.6 \pm 14.1$ & 0.503 & 0.585 & 0.887 & 0.279 \\
\hline \multicolumn{11}{|l|}{ SF-12 score } \\
\hline Physical functioning & $32.5 \pm 12.8$ & $31.5 \pm 9.6$ & $33.3 \pm 11.0$ & $34.4 \pm 12.9$ & $35.5 \pm 10.8$ & $35.5 \pm 11.4$ & 0.853 & 0.816 & 0.326 & 0.905 \\
\hline Role-physical & $21.4 \pm 3.0$ & $20.9 \pm 4.5$ & $21.3 \pm 2.8$ & $22.3 \pm 3.4$ & $22.9 \pm 3.8$ & $22.3 \pm 3.5$ & 0.825 & 0.719 & 0.845 & 0.819 \\
\hline Bodily pain & $33.7 \pm 11.4$ & $32.6 \pm 12.1$ & $36.4 \pm 11.5$ & $37.6 \pm 12.5$ & $40.4 \pm 12.9$ & $39.6 \pm 13.7$ & 0.447 & 0.668 & 0.822 & 0.525 \\
\hline General health & $42.1 \pm 10.0$ & $41.6 \pm 10.5$ & $43.0 \pm 9.7$ & $33.2 \pm 11.9$ & $36.6 \pm 14.0$ & $36.9 \pm 15.1$ & 0.847 & 0.606 & 0.860 & 0.542 \\
\hline Vitality & $44.6 \pm 14.4$ & $40.2 \pm 12.9$ & $45.6 \pm 15.3$ & $45.6 \pm 14.8$ & $45.4 \pm 14.6$ & $43.8 \pm 14.8$ & 0.326 & 0.905 & 0.853 & 0.816 \\
\hline Social functioning & $36.3 \pm 14.6$ & $36.3 \pm 14.2$ & $38.4 \pm 15.1$ & $37.9 \pm 15.8$ & $36.7 \pm 14.1$ & $35.8 \pm 11.7$ & 0.845 & 0.819 & 0.825 & 0.719 \\
\hline Role-emotional & $17.1 \pm 5.2$ & $16.4 \pm 5.3$ & $16.4 \pm 5.1$ & $17.2 \pm 5.1$ & $18.6 \pm 5.1$ & $17.9 \pm 5.4$ & 0.822 & 0.525 & 0.447 & 0.668 \\
\hline Mental health & $40.9 \pm 13.1$ & $43.7 \pm 11.0$ & $41.3 \pm 12.1$ & $40.1 \pm 10.9$ & $43.2 \pm 9.6$ & $43.2 \pm 11.6$ & 0.860 & 0.542 & 0.847 & 0.606 \\
\hline \multicolumn{11}{|l|}{ QST } \\
\hline $\mathrm{CDT},{ }^{\circ} \mathrm{C}$ & $6.6 \pm 12.9$ & $17.8 \pm 12.1$ & $16.6 \pm 11.2$ & $18.1 \pm 11.9$ & $20.3 \pm 10.1$ & $16.4 \pm 12.8$ & 0.906 & 0.767 & 0.679 & 0.468 \\
\hline WDT, ${ }^{\circ} \mathrm{C}$ & $40.8 \pm 6.5$ & $40.1 \pm 6.4$ & $41.9 \pm 6.4$ & $42.9 \pm 5.9$ & $39.5 \pm 5.7$ & $40.0 \pm 6.4$ & $0.001^{d}$ & $<0.001^{d}$ & 0.345 & $0.006^{d}$ \\
\hline $\mathrm{CPT},{ }^{\circ} \mathrm{C}$ & $7.0 \pm 8.7$ & $7.4 \pm 9.2$ & $5.5 \pm 7.7$ & $7.7 \pm 8.6$ & $5.9 \pm 6.8$ & $7.3 \pm 8.7$ & 0.441 & 0.864 & 0.197 & 0.0326 \\
\hline HPT, ${ }^{\circ} \mathrm{C}$ & $47.2 \pm 3.5$ & $47.4 \pm 3.3$ & $47.6 \pm 2.1$ & $48.9 \pm 2.1$ & $47.2 \pm 3.3$ & $46.6 \pm 3.5$ & $0.025^{c}$ & $0.014^{c}$ & 0.703 & $0.029^{c}$ \\
\hline
\end{tabular}

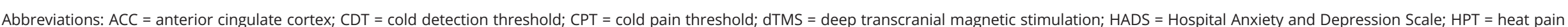
threshold; PCS = Pain Catastrophizing Scale; PSI = posterior superior insula; QST = quantitative sensory testing; SF-12 = Health Survey Quality of Life Questionnaire; WDT = warm detection threshold.

Values are presented as mean $\pm \mathrm{SD}$.

${ }^{a}$ Kruskal Wallis test between the 3 groups.

${ }^{\mathrm{b}}$ Mann-Whitney for changes in scores between groups in pairwise comparisons.

c Significance set at $p<0.05$ after Kruskal-Wallis for differences in the change in score among the 3 groups.

d $p<0.0125$ for Bonferroni correction. 
side effects such as seizures, syncope, falls, persistent headaches, and transient or persistent new neurologic deficits did not occur.

\section{Blinding assessment}

At the end of the study, only $40.6 \%$ of the participants in the PSI-dTMS reported that they were able to tell which group they were allocated to, and among them, $61.5 \%$ guessed it right. In the ACC-dTMS group, these proportions were $62.6 \%$ and $60.0 \%$; and in the sham-dTMS group, $61.2 \%$ and $57.0 \%$, respectively. When the patients were asked if they would like to maintain the sessions of dTMS for a longer period, should this option be offered to them, affirmative answers were given by $62.5 \%$ of the PSI, $67.7 \%$ of the ACC, and $64.5 \%$ of the sham-dTMS patients. These proportions were not significantly different.

\section{Discussion}

The experience of pain is a percept constructed by the interaction of nociceptive ascending inputs with corresponding motivational-emotional and cognitive-evaluative appraisal. Previous attempts to control neuropathic pain by rTMS were aimed mainly at M1 stimulation, and the efficacy of repetitive stimulation sessions has not yet been determined. In a recent guideline, $^{36}$ this limitation has been acknowledged, and the performance of longer studies with multiple maintenance sessions has been supported. Two important deep cortical areas emerge as playing an important role in the central integration of physiologic pain and in the maintenance of central pain: the ACC and the PSI. The ACC is part of the salience network (core control) ${ }^{37}$ and is functionally connected to the anterior insula, being responsible for switching between large-scale networks to facilitate access to attention of salient stimuli, thus integrating sensory inputs to motor, motivational, and mood response, playing a crucial role in the advent of the affective aspects of pain ${ }^{20,38}$ The PSI is a major recipient of spinothalamic inputs to the $\operatorname{cortex}^{39}$ and is thought to participate in the discrimination of painful and nonpainful (e.g., cold) inputs. Functional neuroimaging studies in humans have shown that insula neurons are activated by acute or chronic pain states, ${ }^{40}$ and data from rodent models have revealed that insular neurons can be activated by electric and chemical nociceptive inputs. Direct electric activation of the posterior insula triggers painful sensations in humans, and lesions to the insula reduce pain perception in different body areas. In addition, opioids cause depression of excitatory propagation in the insular cortex in rodents, and insula deep brain stimulation led to opioid- and cannabinoiddependent analgesic effects in a rat model of peripheral neuropathic pain. ${ }^{19}$ It has been proposed that the insula has a tonic hyperalgesic effect, with glutamatergic projections to the ipsilateral amygdala and to GABAergic brainstem neurons projecting to descending modulatory nuclei in the brainstem. Thus, its activation during physiologic conditions would couple the aversive component of the nociceptive stimulus with a loss of top-down discriminatory output to the spinal cord, which would gate its tonic inhibitory inputs to ascending somatosensory inputs (i.e., loss of discrimination, loss of descending inhibition; pronociceptive). In summary, this model proposes that the insula possesses a pronociceptive role when hyperactive, such as in cases of CNP, and local lesions, local injection of opioids, or high-frequency stimulation by deep brain stimulation ${ }^{19,41}$ would decrease its activity and prompt antinociceptive behavior. In this model, the ACC would also be hyperactive in neuropathic pain and would be an important hub in the integration of the affective-emotional components of neuropathic pain. ${ }^{16}$ Here, we have tested the hypothesis that deep TMS of the disinhibited/overactive posterior insula or the ACC by high-frequency stimulations would provide frequency overriding the effects of the corresponding networks, leading to pain relief due to functional inhibition of these structures.

In the present study, we exclusively used deep rTMS. In all groups, stimulation intensity was based on the determination of motor threshold from the leg representation within M1. This approach is frequently used as the best estimation of the electromagnetic field intensity required to reach the deeply located target, because the leg area is deeply located within the hemispheres, $\approx 4$ to $5 \mathrm{~cm}$ from the scalp. In fact, in a recent study, ${ }^{42}$ investigators performed theta-burst stimulation of the PSI cortex by TMS using the double-cone coil using the same protocol as ours and found similar effects on heat pain thresholds in healthy volunteers. In this same study, the authors also measured the distance from the scalp to the PSI and from the scalp from the leg area of M1 for each participant individually and found results similar to ours. ${ }^{25}$ The ACC cortex was aimed at by the use of an $\mathrm{H}$ coil, similar to what has been reported in other studies and replicated. ${ }^{30}$ Recently, dTMS using this same strategy has been granted US Food and Drug Administration approval for obsessive-compulsive disorder treatment. One major point attesting that this strategy actually reaches the PSI and ACC cortices and provides biological effects is that we did find antinociceptive effects only in the PSI arm, and a significant effect on anxiety scores was obtained in the ACC group.

In summary, our results showed that the effects of both active dTMS arms on the clinical pain-related outcomes of the study were all not significantly different from sham stimulation. However, despite the absent effect on the clinical pain in $\mathrm{CPN}$, we have detected a significant antinociceptive effect of insular stimulation compared to sham stimulation, of a magnitude similar to what has been reported after direct stimulation of the insular cortex during stereo-EEG performed before epilepsy surgery ${ }^{41}$ and in rats in a model of neuropathic pain and optogenetics-based ACC stimulation and posterior insular lesion. ${ }^{20} \mathrm{We}$ found a significant increase in warm detection and heat pain thresholds over the painful area after PSI stimulation, which were true antinociceptive/antiallodynic effects (increase in threshold). This finding is superimposable to previous investigators ${ }^{41}$ who described thermal heat 
antinociceptive effects after direct insular high-frequency stimulation (i.e., functional inhibition) in humans under stereo-EEG for surgical epilepsy mapping. This is further evidence suggesting that the present neuronavigated noninvasive stimulation protocol was accurate in its insular targeting. We have also found a significant anxiolytic effect of ACC stimulation in patients with CNP compared to sham stimulation, which was dissociated to the effects on clinical pain relief. Our findings in humans are of striking resemblance to those of an experimental study in which neuropathic pain was induced in mice in which lesions to the ACC reduced exclusively anxiodepressive behaviors related to pain but did not influence its associated abnormal sensory mechanical thresholds. Then, lesions to the posterior insula were performed, and all mood-related behaviors occurring as a result of neuropathic pain remained present ${ }^{20}$ despite changes in mechanical thresholds. The authors also showed that optogenetic-based $^{43}$ stimulation of the ACC at a high frequency $(20 \mathrm{~Hz})$ was able to induce anxiety behaviors in naive rats, supporting the idea that pain can change (i.e., increase) the baseline activity of specific cortical areas and that lesions (or high-frequency stimulation) to these areas can affect the different dimensions of the pain experience differently and specifically.

In the PSI group, the right hemisphere was chosen for several reasons. In one of the largest studies of thalamic CPSP, rightsided infarctions were more commonly associated with CPSP than left-sided infarctions, a finding believed to be consistent with evidence that the right hemisphere is important in pain mediation and body image representation. ${ }^{44}$ In fact, rightsided posterior insular electric stimulations were more frequently associated with evoked pain in humans compared to the left side. A cumulating body of evidence links the right insula to a main role in large-scale switching, and recent evidence has found this structure also to drive altered network disruption in patients with chronic pain. ${ }^{18}$ In addition, left insula activation is related to potential bradycardia, and its high-frequency stimulation could potentially generate language-related side effects. ${ }^{45}$ Notably, both the insula and ACC have neurons with large receptive fields, and one could hypothesize that analgesic effects would occur bilaterally, as has been reported for unilateral M1 stimulation in healthy participants and patients with chronic pain. Unilateral ACC stimulation has indeed triggered bilateral analgesic effects in patients with fibromyalgia, ${ }^{46}$ and insular stimulation during stereo-EEG triggered bilateral sensory responses on direct electric insular stimulation; both high- and low-frequency direct electric stimulation of the superior aspect of the middle short gyrus triggered bilateral pain in the craniofacial region, the throat, and the upper limbs, spreading to the chest and shoulders bilaterally. ${ }^{47}$ Patients with CPSP frequently have pain in large areas of the body, or, as in the case of SCI-related neuropathic pain, symptoms are frequently either axially located (dorsum or lower back or are in fact bilateral). ${ }^{48,49}$ For these reasons, we hypothesized that rightsided insular stimulation would provide diffuse and bilateral effects in patients with both types of CPN. Changes in warm and heat pain thresholds after PSI-dTMS occurred in the pain area, regardless of the side of pain, which further supports our results.

In the present study, we have included patients with different etiologies of CNP. Several studies have demonstrated that the mechanisms and pathophysiology behind neuropathic pain do not cluster according to its etiology (i.e., diabetic polyneuropathy). Instead, it has been shown that a single etiology may cause neuropathic pain of different mechanisms, and different etiologies may share the same mechanisms. Even meta-analyses of the placebo effect in CNP trials have demonstrated that placebo responses are not influenced by the etiology of CNP or its severity. In addition, we performed secondary analyses searching for etiology-based responses (table e-3 available from Dryad, doi.org/10.5061/ dryad.630mg39). We found that both PSI-dTMS and ACCdTMS were not superior to sham stimulation even when stratifying patients by type of CNP (i.e., CPSP and SCI pain).

Concerning other aspects of the trial, we found that dTMS to the ACC and PSI was safe, with no major side effects except for the significant increase in paroxysmal symptoms of neuropathic pain mentioned above in the PSI group. We also found a significant placebo effect in this trial. Large sham effects are relatively common in TMS studies for chronic pain and major depression. Recent data suggest that patients with CNP have significant placebo responses, regardless of the etiology of the lesion (stroke, SCI) or the type of analgesic therapeutic intervention used. Our blinding assessment showed that participants were effectively blinded to their experimental arm, and allocation concealment was well performed. In addition, approximately one-third of the participants were allocated to the sham arms, which comprised 2 types of sham stimulation: one for the ACC and another for the PSI, because these dTMS techniques use different coils. We found no significant differences between the placebo effect of these 2 sham procedures. A similar lack of difference of sham noninvasive cortical stimulation techniques has been reported previously in the only other head-tohead noninvasive neuromodulation published to date for chronic pain relief. ${ }^{32}$

High-frequency dTMS to the (right) PSI or to the ACC has no clinically significant effects compared to sham in CNP, at least when used separately. However, these interventions provided significant antinociceptive (PSIdTMS) and anxiolytic (ACC-dTMS) effects, proving an active biological effect of the stimulation and supporting their targeting accuracy. This information not only may be useful in the design of future noninvasive stimulation paradigms for CNP but also may provide insights into the physiopathology of CNP, a prevalent and refractory form of chronic pain that still lacks evidence-based interventions for its control. 


\section{Author contributions}

Study design and conceptualization: D. Ciampi de Andrade, M.J. Teixeira, R. Galhardoni Geront, L.T. Yeng. Major role in data acquisition: V. Silva, F. Valério, C. Dale, A.F. Baptista, L.M. Barbosa, L.M.B. Menezes, A.M.B.L. Fernandes, F.E.F. da Silva, J. Rosi, A.L. de Lima Rodrigues, P.M. Lorencini Selingardi, D.T. Reis Mendes Fernandes, MA Marcolin. Interpreted data: D. Ciampi de Andrade, R. Galhardoni Geront, C.A. Buchpiguel, C.R. Ono, F.L.S. Duran. Drafted the manuscript for intellectual content: R. Galhardoni, S.R.D.T. de Siqueira, Revised manuscript for intellectual content: D. Ciampi de Andrade, L. García-Larrea, A.R. Brunoni, L.T. Lucato.

\section{Acknowledgment}

The authors thank Sandra Falcon for her technical assistance.

\section{Study funding}

The study was partially funded by Brainsway (Jerusalem, Israel) via a researcher-initiated research grant and by the Pain Center, University of São Paulo. D. Ciampi de Andrade and M.J. Teixeira received $\mathrm{CNPq}$ scholarship for research productivity.

\section{Disclosure}

The authors report no disclosures relevant to the manuscript. Go to Neurology.org/ $\mathrm{N}$ for full disclosures.

\section{Publication history}

Received by Neurology September 25, 2018. Accepted in final form January 14, 2019.

\section{References}

1. Vartiainen N, Perchet C, Magnin M, et al. Thalamic pain: anatomical and physiological indices of prediction. Brain 2016;139:708-722.

2. Siddall PJ, McClelland JM, Rutkowski SB, Cousins MJ. A longitudinal study of the prevalence and characteristics of pain in the first 5 years following spinal cord injury. Pain 2003;103:249-257.

3. Jungehulsing GJ, Israel $\mathrm{H}$, Safar $\mathrm{N}$, et al. Levetiracetam in patients with central neuropathic post-stroke pain: a randomized, double-blind, placebo-controlled trial. Eur J Neurol 2013;20:331-337.

4. Kim JS, Bashford G, Murphy TK, Martin A, Dror V, Cheung R. Safety and efficacy of pregabalin in patients with central post-stroke pain. Pain 2011;152:1018-1023.

5. Vranken JH, Hollmann MW, van der Vegt MH, et al. Duloxetine in patients with central neuropathic pain caused by spinal cord injury or stroke: a randomized, doubleblind, placebo-controlled trial. Pain 2011;152:267-273.

6. Attal N, Guirimand F, Brasseur L, Gaude V, Chauvin M, Bouhassira D. Effects of IV morphine in central pain: a randomized placebo-controlled study. Neurology 2002; 58:554-563.

7. Leijon G, Boivie J. Central post-stroke pain: a controlled trial of amitriptyline and carbamazepine. Pain 1989;36:27-36.

8. Richards JS, Bombardier CH, Wilson CS, et al. Efficacy of venlafaxine XR for the treatment of pain in patients with spinal cord injury and major depression: a randomized, controlled trial. Arch Phys Med Rehabil 2015;96:680-689.

9. Falah M, Madsen C, Holbech JV, Sindrup SH. A randomized, placebo-controlled trial of levetiracetam in central pain in multiple sclerosis. Eur J Pain 2012;16: $860-869$.

10. Rintala DH, Fiess RN, Tan G, Holmes SA, Bruel BM. Effect of dronabinol on central neuropathic pain after spinal cord injury: a pilot study. Am J Phys Med Rehabil 2010; 89:840-848.

11. Langford RM, Mares J, Novotna A, Vachova I, Notcutt W, Ratcliffe S. A doubleblind, randomized, placebo-controlled, parallel-group study of THC/CBD oromucosal spray in combination with the existing treatment regimen, in the relief of central neuropathic pain in patients with multiple sclerosis. J Neurol 2013;260: 984-997.

12. Vollmer TL, Robinson MJ, Risser RC, Malcolm SK. A randomized, double-blind, placebo-controlled trial of duloxetine for the treatment of pain in patients with multiple sclerosis. Pain Pract 2014;14:732-744.
13. Barrera-Chacon JM, Mendez-Suarez JL, Jauregui-Abrisqueta ML, Palazon R, BarbaraBataller E, Garcia-Obrero I. Oxycodone improves pain control and quality of life in anticonvulsant-pretreated spinal cord-injured patients with neuropathic pain. Spinal Cord 2011;49:36-42.

14. Hosomi K, Shimokawa T, Ikoma K, et al. Daily repetitive transcranial magnetic stimulation of primary motor cortex for neuropathic pain: a randomized multicenter, double-blind, crossover, sham-controlled trial. Pain 2013;154: 1065-1072.

15. de Oliveira RA, de Andrade DC, Machado AG, Teixeira MJ. Central poststroke pain: somatosensory abnormalities and the presence of associated myofascial pain syndrome. BMC Neurol 2012;12:89.

16. Lieberman MD, Eisenberger NI. The dorsal anterior cingulate cortex is selective for pain: results from large-scale reverse inference. Proc Natl Acad Sci USA 2015;112: $15250-15255$.

17. Peyron R, Laurent B, Garcia-Larrea L. Functional imaging of brain responses to pain: a review and meta-analysis (2000). Neurophysiol Clin 2000;30: 263-288.

18. Cottam WJ, Iwabuchi SJ, Drabek MM, Reckziegel D, Auer DP. Altered connectivity of the right anterior insula drives the pain connectome changes in chronic knee osteoarthritis. Pain 2018;159:929-938.

19. Dimov LF, Toniolo EF, Alonso-Matielo H, et al. Electrical stimulation of the insular cortex as a novel target for the relief of refractory pain: an experimental approach in rodents. Behav Brain Res 2018;346:86-95.

20. Barthas F, Sellmeijer J, Hugel S, Waltisperger E, Barrot M, Yalcin I. The anterior cingulate cortex is a critical hub for pain-induced depression. Biol Psychiatry 2015;77: 236-245.

21. Carmi L, Alyagon U, Barnea-Ygael N, Zohar J, Dar R, Zangen A. Clinical and electrophysiological outcomes of deep TMS over the medial prefrontal and anterior cingulate cortices in OCD patients. Brain Stimul 2018;11:158-165.

22. Finnerup NB, Haroutounian S, Kamerman P, et al. Neuropathic pain: an updated grading system for research and clinical practice. Pain 2016;157: 1599-1606.

23. Ciampi de Andrade D, Galhardoni R, Pinto LF, Lancelotti J, Marcolin MA, Teixeira MJ. Into the island: a new technique of non-invasive cortical stimulation of the insula. Neurophysiol Clin 2012;42:363-368.

24. Deng ZD, Lisanby SH, Peterchev AV. Electric field depth-focality tradeoff in transcranial magnetic stimulation: simulation comparison of 50 coil designs. Brain Stimul 2013;6:1-13.

25. Andre-Obadia N, Mertens P, Gueguen A, Peyron R, Garcia-Larrea L. Pain relief by rTMS: differential effect of current flow but no specific action on pain subtypes. Neurology 2008;71:833-840.

26. Kranz G, Shamim EA, Lin PT, Kranz GS, Hallett M. Transcranial magnetic brain stimulation modulates blepharospasm: a randomized controlled study. Neurology 2010;75:1465-1471.

27. Levkovitz Y, Isserles M, Padberg F, et al. Efficacy and safety of deep transcranial magnetic stimulation for major depression: a prospective multicenter randomized controlled trial. World Psychiatry 2015;14:64-73.

28. Dinur-Klein L, Dannon P, Hadar A, et al. Smoking cessation induced by deep repetitive transcranial magnetic stimulation of the prefrontal and insular cortices: a prospective, randomized controlled trial. Biol Psychiatry 2014;76: 742-749.

29. de Andrade DC, Ferreira KA, Nishimura CM, et al. Psychometric validation of the Portuguese version of the Neuropathic Pain Symptoms Inventory. Health Qual Life Outcomes 2011;9:107.

30. Cury RG, Galhardoni R, Teixeira MJ, et al. Subthalamic deep brain stimulation modulates conscious perception of sensory function in Parkinson's disease. Pain 2016;157:2758-2765.

31. Cueva AS, Galhardoni R, Cury RG, et al. Normative data of cortical excitability measurements obtained by transcranial magnetic stimulation in healthy subjects. Neurophysiol Clin 2016;46:43-51.

32. Attal N, Ayache SS, Ciampi De Andrade D, et al. Repetitive transcranial magnetic stimulation and transcranial direct-current stimulation in neuropathic pain due to radiculopathy: a randomized sham-controlled comparative study. Pain 2016;157: 1224-1231.

33. Templ M, Alfons A, Kowarik A, and Prantner B. VIM: Visualization and Imputation of Missing Values, 2011a. Available at: http://CRAN.R-project.org/package=VIM. $\mathrm{R}$ package version 3.0.0. Accessed March 23, 2019.

34. Allaire JCJ, Xie Y, McPherson J, et al. Rmarkdown: dynamic documents for $\mathrm{R}$ [online]. Available at: CRAN.R-project.org/package $=$ rmarkdown. Accessed May, 2016

35. Mhalla A, Baudic S, Ciampi de Andrade D, et al. Long-term maintenance of the analgesic effects of transcranial magnetic stimulation in fibromyalgia. Pain 2011;152: $1478-1485$

36. Cruccu G, Garcia-Larrea L, Hansson P, et al. EAN guidelines on central neurostimulation therapy in chronic pain conditions. Eur J Neurol 2016;23: 1489-1499.

37. Menon V, Uddin LQ. Saliency, switching, attention and control: a network model of insula function. Brain Struct Funct 2010;214:655-667.

38. Fuchs PN, Peng YB, Boyette-Davis JA, Uhelski ML. The anterior cingulate cortex and pain processing. Front Integr Neurosci 2014;8:35.

39. Dum RP, Levinthal DJ, Strick PL. The spinothalamic system targets motor and sensory areas in the cerebral cortex of monkeys. J Neurosci 2009;29: $14223-14235$. 
Ploghaus A, Tracey I, Gati JS, Clare RS, Matthews PM, Rawlins JN. Dissociating pain from its anticipation in the human brain. Science 1999;284: 1979-1981.

41. Denis DJ, Marouf R, Rainville P, Bouthillier A, Nguyen DK. Effects of insular stimulation on thermal nociception. Eur J Pain 2016;20:800-810.

42. Lenoir C, Algoet M, Mouraux A. Deep continuous theta burst stimulation of the operculo-insular cortex selectively affects Adelta-fibre heat pain. J Physiol 2018;596: 4767-4787.

43. Benison AM, Chumachenko S, Harrison JA, Maier SP, Watkins LR, Barth DS. Caudal granular insular cortex is sufficient and necessary for the long-term maintenance of allodynic behavior in the rat attributable to mononeuropathy. J Neurosci 2011;31: 6317-6328.

44. Nasreddine ZS, Saver JL. Pain after thalamic stroke: right diencephalic predominance and clinical features in 180 patients. Neurology 1997;48:1196-1199.
45. Lorca-Puls DL, Gajardo-Vidal A, Seghier ML, et al. Using transcranial magnetic stimulation of the undamaged brain to identify lesion sites that predict language outcome after stroke. Brain 2017;140:1729-1742.

46. Tzabazis A, Aparici CM, Rowbotham MC, Schneider MB, Etkin A, Yeomans DC Shaped magnetic field pulses by multi-coil repetitive transcranial magnetic stimulation (rTMS) differentially modulate anterior cingulate cortex responses and pain in volunteers and fibromyalgia patients. Mol Pain 2013;9:33.

47. Afif A, Hoffmann D, Minotti L, Benabid AL, Kahane P. Middle short gyrus of the insula implicated in pain processing. Pain 2008;138:546-555.

48. Martinelli Boneschi F, Colombo B, Annovazzi P, et al. Lifetime and actual prevalence of pain and headache in multiple sclerosis. Mult Scler 2008;14: 514-521.

49. Nurmikko TJ, Gupta S, Maclver K. Multiple sclerosis-related central pain disorders. Curr Pain Headache Rep 2010;14:189-195. 


\section{Neurology}

\section{Insular and anterior cingulate cortex deep stimulation for central neuropathic pain: Disassembling the percept of pain}

Ricardo Galhardoni, Valquíria Aparecida da Silva, Luís García-Larrea, et al. Neurology 2019;92;e2165-e2175 Published Online before print April 5, 2019

DOI 10.1212/WNL.0000000000007396

\section{This information is current as of April 5, 2019}

\section{Updated Information \&} Services

References

Citations

Subspecialty Collections

Permissions \& Licensing

Reprints including high resolution figures, can be found at: http://n.neurology.org/content/92/18/e2165.full

This article cites 47 articles, 8 of which you can access for free at: http://n.neurology.org/content/92/18/e2165.full\#ref-list-1

This article has been cited by 2 HighWire-hosted articles: http://n.neurology.org/content/92/18/e2165.full\#\#otherarticles

This article, along with others on similar topics, appears in the following collection(s):

All CBMRT/Null Hypothesis

http://n.neurology.org/cgi/collection/all_cbmrt_null_hypothesis

Central pain

http://n.neurology.org/cgi/collection/central_pain

Neuropathic pain

http://n.neurology.org/cgi/collection/neuropathic_pain TMS

http://n.neurology.org/cgi/collection/tms

Information about reproducing this article in parts (figures,tables) or in its entirety can be found online at:

http://www.neurology.org/about/about_the_journal\#permissions

Information about ordering reprints can be found online:

http://n.neurology.org/subscribers/advertise

Neurology ${ }^{\circledR}$ is the official journal of the American Academy of Neurology. Published continuously since 1951, it is now a weekly with 48 issues per year. Copyright @ 2019 American Academy of Neurology. All rights reserved. Print ISSN: 0028-3878. Online ISSN: 1526-632X.

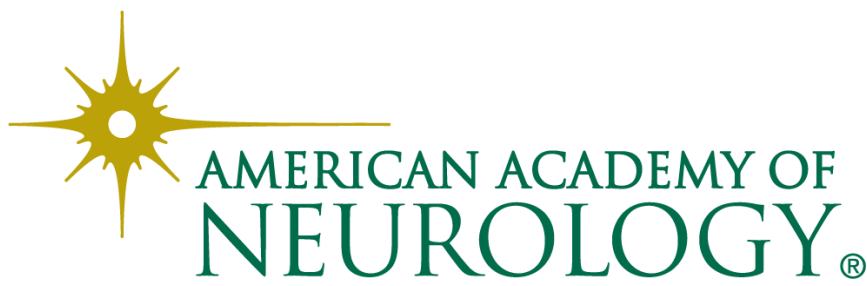

